\title{
Operation of the Metrology Light Source as a primary radiation source standard
}

\author{
Roman Klein, Guido Brandt, Rolf Fliegauf, Arne Hoehl, Ralph Müller, Reiner Thornagel, and Gerhard Ulm \\ Physikalisch-Technische Bundesanstalt, Berlin, Germany
}

Michael Abo-Bakr, Jörg Feikes, Michael v. Hartrott, Karsten Holldack, and Godehard Wüstefeld

BESSY, Berlin, Germany

(Received 26 August 2008; published 20 November 2008)

\begin{abstract}
The Metrology Light Source (MLS), the new electron storage ring of the Physikalisch-Technische Bundesanstalt (PTB) located in Berlin, is dedicated to metrology and technological developments in the UV and extreme UV spectral range as well as in the IR and THz region. The MLS can be operated at any electron beam energy between 105 and $630 \mathrm{MeV}$ and at electron beam currents varying from $1 \mathrm{pA}$ (one stored electron) up to $200 \mathrm{~mA}$. Moreover, it is optimized for the generation of coherent synchrotron radiation in the far IR/THz range. Of special interest for PTB is the operation of the MLS as a primary radiation source standard from the near IR up to the soft x-ray region. Therefore, the MLS is equipped with all the instrumentation necessary to measure the storage ring parameters and geometrical parameters needed for the calculation of the spectral photon flux according to the Schwinger theory with low uncertainty.
\end{abstract}

DOI: 10.1103/PhysRevSTAB.11.110701

PACS numbers: 06.20.F-, 07.85.Fv, 07.85.Qe

\section{INTRODUCTION}

Electron storage rings with calculable bending magnet radiation according to the Schwinger theory are used as primary source standards for radiometry at several national metrology institutes [1]. Since the spectral range covered by electron storage rings extends far into the x-ray region, their usage for radiometry considerably expands the spectral region as compared to that covered by blackbody radiators, which are routinely used for conventional radiometry from the IR to the UV region. For more than 25 years, the Physikalisch-Technische Bundesanstalt (PTB) has been taking advantage of this [2], at the nowclosed BESSY I electron storage ring, at BESSY II, and now at the Metrology Light Source (MLS), which is located next to BESSY II in Berlin-Adlershof. Figure 1 illustrates the tremendous expansion of the spectral range for the primary synchrotron radiation sources with different characteristic photon energies $E_{c}$ used by PTB as compared to a blackbody radiator. The option of altering the synchrotron radiation spectrum, i.e. by choosing different characteristic energies, is important, e.g. in order to suppress higher diffraction orders from monochromators or to reduce the thermal load on optical components.

The major applications are the calibration of radiation sources and energy-dispersive detectors. Radiation sources are calibrated by comparing their unknown spectral photon flux with the calculable synchrotron radiation flux [3]. For this purpose, wavelength dispersive experimental stations, e.g. consisting of an imaging mirror, a monochromator, and a detector, are used. Energy-dispersive detectors are calibrated in the direct synchrotron radiation beam while operating the storage ring at very low beam currents, in many cases only with a few stored electrons [4].

\section{ELECTRON STORAGE RINGS AS PRIMARY RADIATION STANDARDS}

A prerequisite to the operation of an electron storage ring as a primary source standard is - in addition to sufficient stability - the knowledge of the parameters needed for the calculation of the spectral radiant power based on

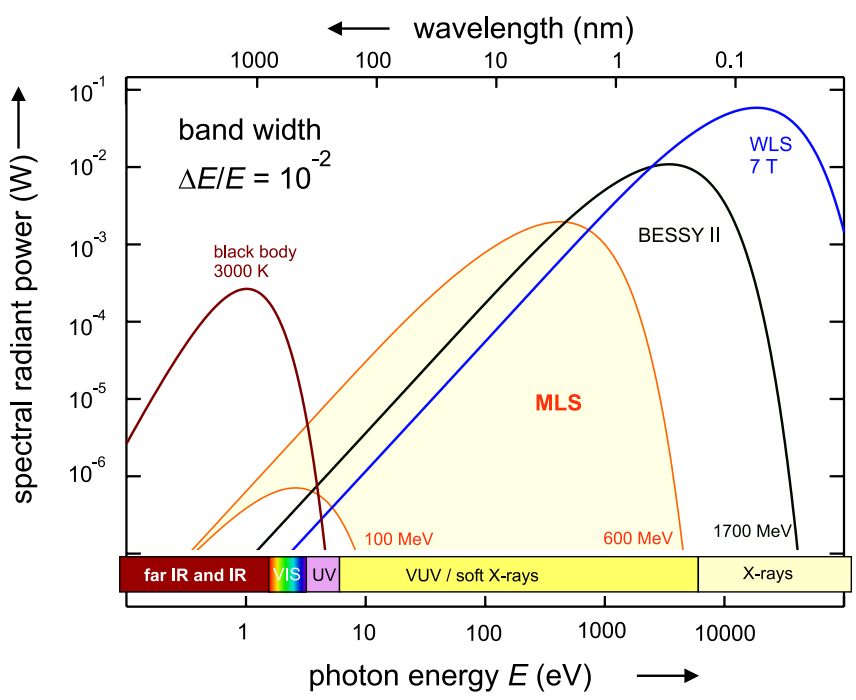

FIG. 1. (Color) Calculated spectral radiant power of various primary synchrotron radiation sources with different characteristic photon energies $E_{c}$ used by PTB as compared to a blackbody radiator for typical angular acceptances. For the synchrotron radiation sources MLS and BESSY II $\left(E_{c}=\right.$ $2.5 \mathrm{keV}$ ), an electron beam current of $200 \mathrm{~mA}$ was assumed. The spectrum of a $7 \mathrm{~T}$ wavelength shifter (WLS, $E_{c}=$ $13.5 \mathrm{keV}$ ), operated at BESSY II and used by PTB for x-ray radiometry, is also shown. 
the Schwinger equation [5]. The electron storage ring BESSY II is operated by PTB as a primary source standard mainly for the x-ray spectral range [6]. The MLS electron storage ring $[7,8]$, which has a lower characteristic energy as compared to BESSY II, will also be operated as a primary source standard and for that reason PTB has installed and is operating all the equipment for the measurement of the storage ring parameters and geometrical quantities needed for the calculation of the spectral photon flux with high accuracy $[9,10]$. The geometrical parameters that define the angular acceptance also have to be determined. The spectral radiant power $\Phi_{E}$ for a photon energy $E$ can then be expressed in the general form as

$$
\Phi_{E}=\Phi_{E}\left(E ; W, B, I, \Sigma_{y} ; \Psi, d, a, b\right) .
$$

The parameters are: the electron energy $W$; the magnetic induction $B$ at the radiation source point; the electron beam current $I$; the effective vertical source size $\Sigma_{y}$; the vertical emission angle $\psi$; the distance $d$ between the radiation source point and a flux-defining aperture of size $a \times b$ (see Fig. 2). The effective vertical source size $\Sigma_{y}$ (or effective vertical source divergence $\Sigma_{y^{\prime}}$ ) at the aperture is derived from the vertical electron beam size $\sigma_{y}$ and beam divergence $\sigma_{y^{\prime}}$ according to $\Sigma_{Y}=\left(\sigma_{y}^{2}+d^{2} \sigma_{y^{\prime}}{ }^{2}\right)^{1 / 2}=d \Sigma_{Y^{\prime}}$.

The calculation of the radiant power is performed numerically as follows.

The Schwinger equation gives the spectral energy per solid angle radiated by one electron of energy $W$ moving on a circular arc with bending radius $R=W / e c B$. In an electron storage ring, many electrons of number $N$ are stored, which revolve in the ring with frequency $\nu$. The spectral power per solid angle (spectral radiant intensity) is then given by multiplying the original Schwinger equation by $\nu N$. The spectral radiant intensity for electrons of beam current $I=e \nu N$ and energy $W$, all moving exactly on a

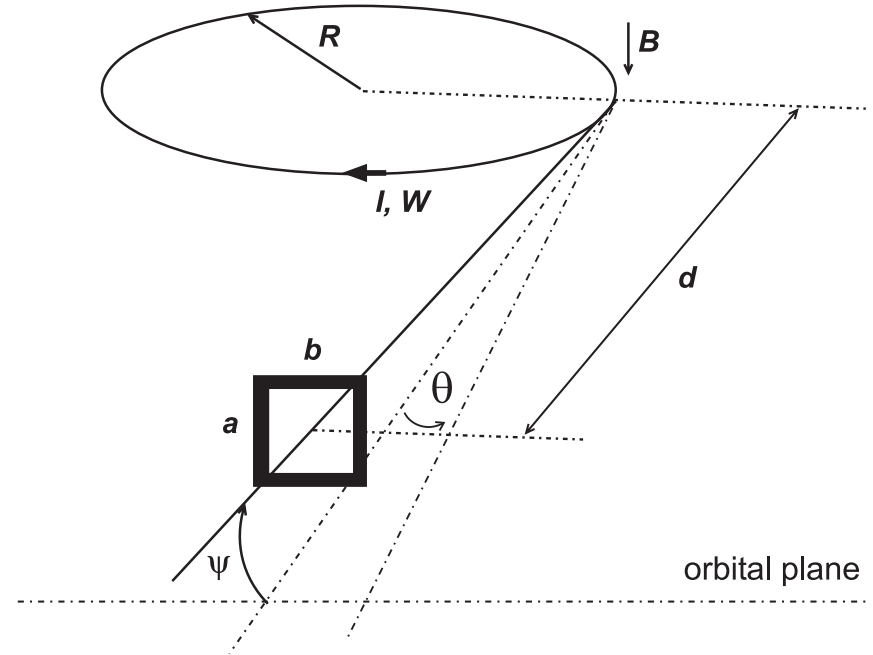

FIG. 2. Geometry and parameters used for the calculation of the radiant power emitted from a bending magnet. circular arc with radius $R$, is given for the $\sigma$ component (electric field vector in the orbital plane) as

$$
\begin{aligned}
I_{0 E}^{\sigma} & =\frac{d}{d E} \frac{d^{2}}{d \theta d \psi} \phi^{\sigma} \\
& =\frac{2 e I R^{2}}{3 \varepsilon_{0} \gamma^{4}} \frac{E^{2}}{(h c)^{3}}\left\{\left[1+(\gamma \psi)^{2}\right]^{2} K_{2 / 3}^{2}(\xi)\right\}
\end{aligned}
$$

and for the $\pi$-polarization component (electric field vector perpendicular to the orbital plane and shifted in phase by $\pi / 2$ with respect to the $\sigma$ component) as

$$
\begin{aligned}
I_{0 E}^{\pi} & =\frac{d}{d E} \frac{d^{2}}{d \theta d \psi} \phi^{\pi} \\
& =\frac{2 e I R^{2}}{3 \varepsilon_{0} \gamma^{4}} \frac{E^{2}}{(h c)^{3}}\left\{\left[1+(\gamma \psi)^{2}\right](\gamma \psi)^{2} K_{1 / 3}^{2}(\xi)\right\}
\end{aligned}
$$

with

$$
\begin{aligned}
& \xi=\frac{2 \pi R E}{3 h c \gamma^{3}}\left[1+(\gamma \psi)^{2}\right]^{3 / 2}=\frac{1}{2} \frac{E}{E_{c}}\left[1+(\gamma \psi)^{2}\right]^{3 / 2} \\
& \gamma=\frac{W}{m_{e} c^{2}} .
\end{aligned}
$$

The $K_{1 / 3}$ and $K_{2 / 3}$ are modified Bessel functions of the second kind, $\theta$ and $\Psi$ are the horizontal and vertical angles with respect to the direction of the electrons. $E_{c}$ is the characteristic photon energy and defined as $E_{c}=\frac{3 \hbar c \gamma^{3}}{2 R}$.

In reality, the stored electrons have a small deviation from the perfect circular orbit with standard widths of $\sigma_{x}$ and $\sigma_{y}$ in space and $\sigma_{x^{\prime}}$ and $\sigma_{y^{\prime}}$ in angle for the horizontal and vertical direction, respectively. The horizontal distribution is of no importance due to the tangential observation of the synchrotron radiation, but in the vertical direction this leads to an effective divergence $\Sigma_{Y^{\prime}}$ at the location of the aperture stop placed at distance $d$, which has to be taken into account as a convolution over the vertical emission angle $\Psi$.

The spectral radiant power through a flux-defining aperture is then calculated as

$$
\begin{aligned}
\Phi_{E}= & \frac{d \Phi}{d E}(E) \\
= & \iint_{\text {aperture }}\left[\int_{-\infty}^{+\infty} \frac{1}{\sqrt{2 \pi} \Sigma_{y^{\prime}}}\left[I_{0 E}^{\sigma}\left(\psi^{\prime \prime}\right)+I_{0 E}^{\pi}\left(\psi^{\prime \prime}\right)\right]\right. \\
& \left.\times e^{-\left(\left(\psi^{\prime \prime}-\psi^{\prime}\right)^{2} / 2 \Sigma_{y^{\prime}}^{2}\right)} d \psi^{\prime \prime}\right] d \psi^{\prime} d \theta .
\end{aligned}
$$

For a rectangular aperture of size $a \times b$ at distance $d$, the integration over the horizontal angle $\theta$ in the orbit plane results in a factor $b / d$. In the perpendicular direction, the integration over the vertical angle $\psi^{\prime}$ has to be performed from $(\psi-a / 2 d)$ up to $(\psi+a / 2 d)$ with $\psi$ being the observation angle of the center of the aperture with respect to the orbit plane $(a / d, b / d, \psi \ll 1)$. 
Besides the fact that the spectrum of electron storage rings covers a wide spectral range and is calculable from fundamental electrodynamics relations, electron storage rings have some other properties, which make them ideal sources for radiometry: The spectral radiant power scales linearly with the stored electron beam, which can be adjusted in a range of up to 12 decades. Moreover, the spectral shape of the emitted spectrum can be adjusted by the variation of the electron beam. Since most electron storage rings are multiuser facilities, these options can rarely be exploited, except at dedicated storage rings (e.g. the MLS) or in special shifts (e.g. special user shifts of PTB at BESSY II). With reduced demands on the achievable uncertainty in the calculation, PTB also uses a superconducting $7 \mathrm{~T}$ WLS installed at BESSY II as a source of calculable radiation [11] in the hard X-ray spectral region.

It should be noted that the above stated linear scaling of the radiant power with the number of stored electrons is only true for wavelengths of the emitted light that are much smaller than the electron bunch length (typically $5 \mathrm{~mm}$ at the MLS in normal operation). For wavelengths in the range of the electron bunch length or longer, the electrons in a bunch can emit coherently, which results in a quadratic scaling with the number of electrons in a bunch. This leads to an enhancement of the emitted power by many orders of magnitude for those wavelengths. Therefore, for the pro- duction of intense $\mathrm{THz}$ radiation, short electron bunch lengths are required, which can be achieved by operation of the electron storage ring in a special low- $\alpha$ mode [12]. The MLS is optimized for this special operation mode and for the production of intense $\mathrm{THz}$ radiation [13].

\section{MEASUREMENT OF THE MLS PARAMETERS}

At the MLS, the electron beam current and electron energy can be varied over a wide range in order to create tailor-made conditions for special applications and calibrations, as can be seen in Table I, which lists the main parameters of the MLS. The instrumentation for the measurement of the storage ring parameters must be suitable to cover that wide range. The uncertainties in the measurement of the parameters determine the uncertainty of the calculation of the spectral radiant power. Table II lists typical values of the MLS parameters and the influence of their uncertainties on the uncertainty of the calculation of the spectral radiant power. The left column lists the parameters with the anticipated uncertainty in the measurement. The right columns list the influence of the uncertainty in these parameters on the relative uncertainty in the calculation of the spectral radiant power for different photon energies. This influence has been calculated numerically by the variation of the input parameters within the range defined by the measurement uncertainty.

TABLE I. Main MLS parameters.

\begin{tabular}{ll}
\hline \hline \multicolumn{1}{c}{ Parameter } & \multicolumn{1}{c}{ Value } \\
\hline Lattice structure & Double bend achromat \\
Circumference & $48 \mathrm{~m}$ \\
Electron energy & 105 to $630 \mathrm{MeV}$ \\
Magnetic induction of bending magnets & 0.23 to $1.38 \mathrm{~T}$ \\
Characteristic wavelength & 3.4 to $735 \mathrm{~nm}$ \\
Characteristic photon energy & 1.7 to $364 \mathrm{eV}$ \\
Electron beam current & $1 \mathrm{pA}$ to $200 \mathrm{~mA}$ \\
Natural emittance (design value at $600 \mathrm{MeV})$ & $100 \mathrm{~nm} \mathrm{rad}$ \\
Injection energy & $105 \mathrm{MeV}$ \\
\hline \hline
\end{tabular}

TABLE II. Parameters that enter the calculation with typical values and uncertainties and their influence on the relative uncertainty in the calculation of the radiant power for different photon energies.

\begin{tabular}{lllcc}
\hline \hline & & \multicolumn{2}{c}{$\Delta \Phi_{E}(E) / \Phi_{E}(E)\left(\times 10^{-3}\right)$ at $E=$} \\
\multicolumn{1}{c}{ Parameter/typical value } & \multicolumn{2}{c}{$1 \mathrm{eV}$} & $100 \mathrm{eV}$ & $1000 \mathrm{eV}$ \\
\hline Electron beam current $(100.00 \pm 0.02) \mathrm{mA}$ & $I$ & 0.20 & 0.20 & 0.20 \\
Electron energy $(600.00 \pm 0.06) \mathrm{MeV}$ & $W$ & 0.07 & 0.12 & 0.67 \\
Magnetic induction $(1.30000 \pm 0.00013) \mathrm{T}$ & $B$ & 0.07 & 0.04 & 0.27 \\
Effective vertical divergence $(44 \pm 9) \mu \mathrm{rad}$ & $\Sigma_{y}$ & 0.04 & 0.18 & 1.5 \\
Distance to source point $(15000 \pm 2) \mathrm{mm}$ & $d$ & 0.27 & 0.26 & 0.17 \\
Angle to orbit plane $(0.000 \pm 0.005) \mathrm{mrad}$ & $\psi$ & 0.0007 & 0.003 & 0.03 \\
Total uncertainty & & 0.35 & 0.40 & 1.7 \\
\hline \hline
\end{tabular}


The setups and results for the determination of the parameters are described in more detail below, with emphasis on the measurement of the electron beam energy and electron beam current, since they require the most sophisticated technique.

\section{A. Electron beam energy}

The electron beam energy can be ramped to any value from $105 \mathrm{MeV}$ (injection energy) up to the maximum energy of $630 \mathrm{MeV}$ and is determined by the method of Compton-backscattering of laser photons [14]. Therefore, a $\mathrm{CO}_{2}$ laser beam is superimposed antiparallel to the electron beam and the photons scattered in the forward direction of the electron beam are measured with an energy-dispersive detector, the channel energy of which has been calibrated by radionuclides. From the cutoff energy $E_{2}^{\max }$ of the spectrum, the electron energy $W=$ $\gamma m_{e} c^{2}$ can be determined according to

$$
E_{2}^{\max }=E_{1} 4 \gamma^{2} \frac{1}{1+4 \gamma^{2} / m_{e} c^{2}}
$$

for head-on collisions and observation of the scattered photons in the direction of the electron beam [15], with $E_{1}$ being the laser photon energy.

The cutoff energy varies from $20 \mathrm{keV}$ up to $710 \mathrm{keV}$ for the MLS operated at 105 and $630 \mathrm{MeV}$, respectively. To cover this wide range, two different detectors have been used as well as a slightly different optical path as is illustrated in Fig. 3: For the high energy end, i.e. electron energies from $630 \mathrm{MeV}$ down to $250 \mathrm{MeV}$, an HPGe coaxial detector with a large crystal is used (EG\&G GEM-100220-P). Because of the large Ge-crystal, this detector has a good efficiency for the detection of high energy photons but is not suited for low-energy photon detection because of its thick Ge-dead layer and thick Alentrance window. This detector system is energy calibrated by using ${ }^{60} \mathrm{Co}$ and natural radioactivity background lines. The backscattered photons penetrate the mirror used to
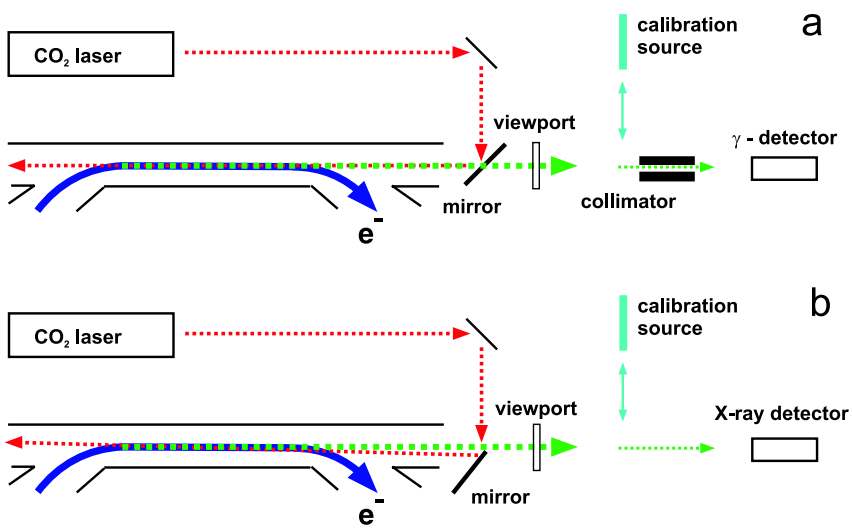

FIG. 3. (Color) Schematic setup of the instrumentation for the measurement of the electron beam energy for high (a) and low (b) electron beam energy values.

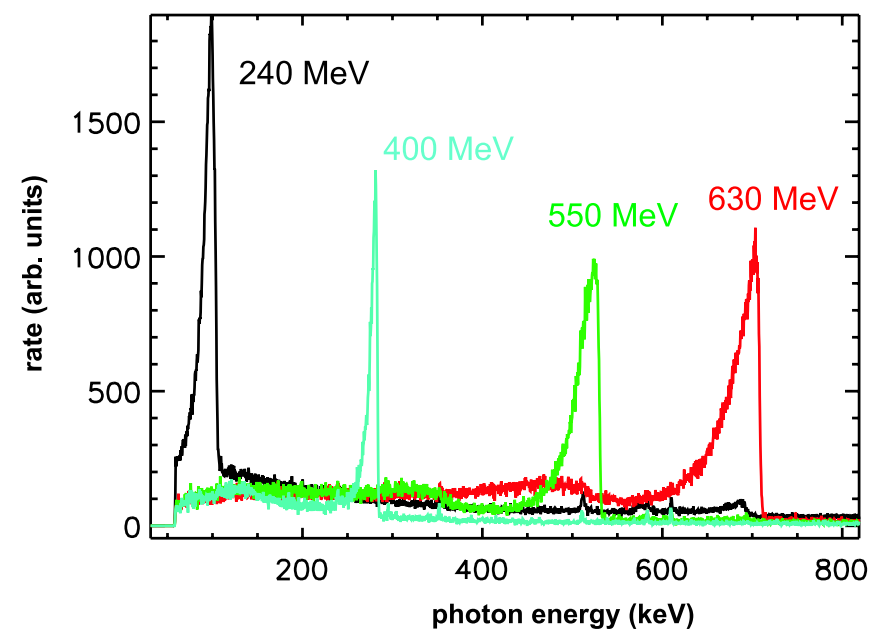

FIG. 4. (Color) Typical spectra of Compton-backscattered photons for high electron beam energies.

superimpose the laser beam [Fig. 3(a)]. A tungsten collimator limits the angular acceptance to the central cone in order not to saturate the detector with undesired lowenergy photons. Figure 4 shows some typical spectra of the backscattered photons for various electron energies. Figure 5 illustrates a close-up of the cutoff edge for the scattered spectrum at $628 \mathrm{MeV}$ operation of the MLS with the edge function (red line) fitted to the data points. The function used to describe the cutoff edge is

$$
\begin{aligned}
f\left(x ; a_{1} \cdots a_{5}\right)= & a_{3}\left\{\left[1+a_{4}\left(x-a_{1}\right)\right]\right. \\
& \times \frac{\operatorname{erfc}\left[\left(x-a_{1}\right) / \sqrt{2} a_{2}\right]}{2} \\
& \left.-\frac{a_{2} a_{4} \exp \left[-\left(x-a_{1}\right)^{2} / 2 a_{2}^{2}\right]}{\sqrt{2 \pi}}\right\}+a_{5}
\end{aligned}
$$

with $\operatorname{erfc}(x)=\frac{2}{\sqrt{\pi}} \int_{x}^{\infty} \exp \left(-t^{2}\right) d t$

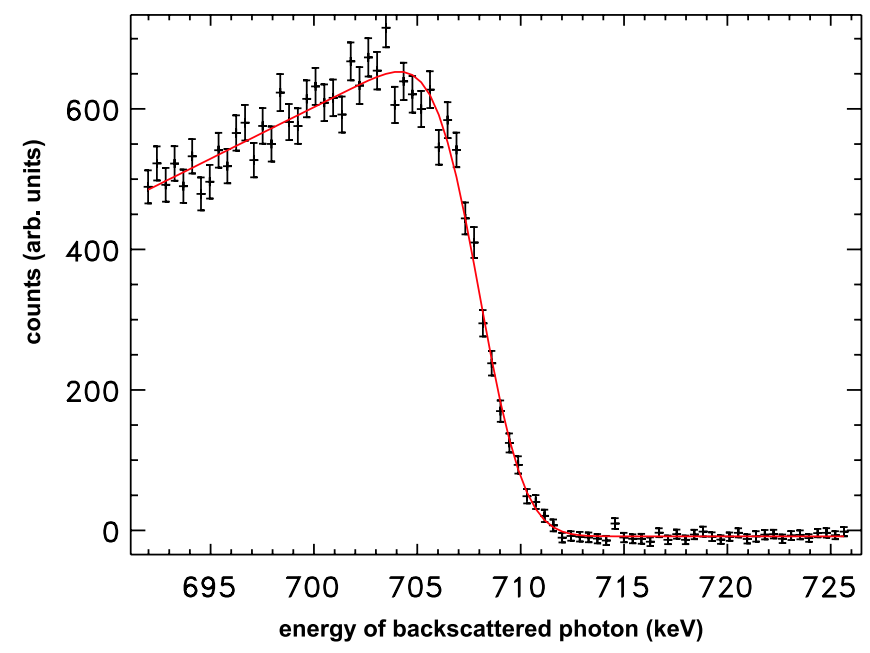

FIG. 5. (Color) Close-up of the cutoff edge of the backscattered photons for $628 \mathrm{MeV}$ operation of the MLS. 
This edge function $f(x)$ is derived as the convolution of a step function

$$
h(x)= \begin{cases}a_{3}\left[1+a_{4}\left(x-a_{1}\right)\right] & \text { for } x \leq a_{1} \\ 0 & \text { else }\end{cases}
$$

which represents the cutoff edge, with a Gaussian function $g(x)=\exp \left(-x^{2} / 2 a_{2}^{2}\right) / \sqrt{2 \pi} a_{2}$, which attributes to the finite detector energy resolution and energy spread of the electron beam.

The fitted parameters $\left(a_{1} \ldots a_{5}\right)$ are the position of the cutoff edge, standard deviation of the folded Gaussian, height of the cutoff edge, slope of the spectrum before the cutoff edge and a constant offset. A relative uncertainty of $1 \times 10^{-4}$ in the determination of the electron energy can be achieved. More details on the data evaluation can be found in Ref. [15].

For the low-energy side of the backscattered photons, i.e. for electron energies of $400 \mathrm{MeV}$ down to $105 \mathrm{MeV}$, a LEGe detector with Be-entrance window and rear contact (Canberra GL 0515) was used. This detector is optimized for the detection of low-energy photons above $3 \mathrm{keV}$ but its efficiency rapidly decreases for photon energies above approximately $100 \mathrm{keV}$. This detector system has been energy calibrated by using ${ }^{57} \mathrm{Co}$. The mirror used to superimpose the laser beam was positioned in such a way to let the scattered photons pass without interaction [Fig. 3(b)]. Nevertheless, the scattered photons have to penetrate a viewport that terminates the vacuum system and that ultimately limits the detection of low-energy photons to approximately $20 \mathrm{keV}$. Typical spectra for this setup are shown in Fig. 6. Measurements in the overlapping region show good agreement for either detector system or optical alignment. In the setup according to Fig. 3(b), no collimator was used, since due to the smaller electron energies the angular divergence of the backscattered photons is much wider and the size of the detector limits the angular acceptance. Figure 7 illustrates the influence of a collimator on

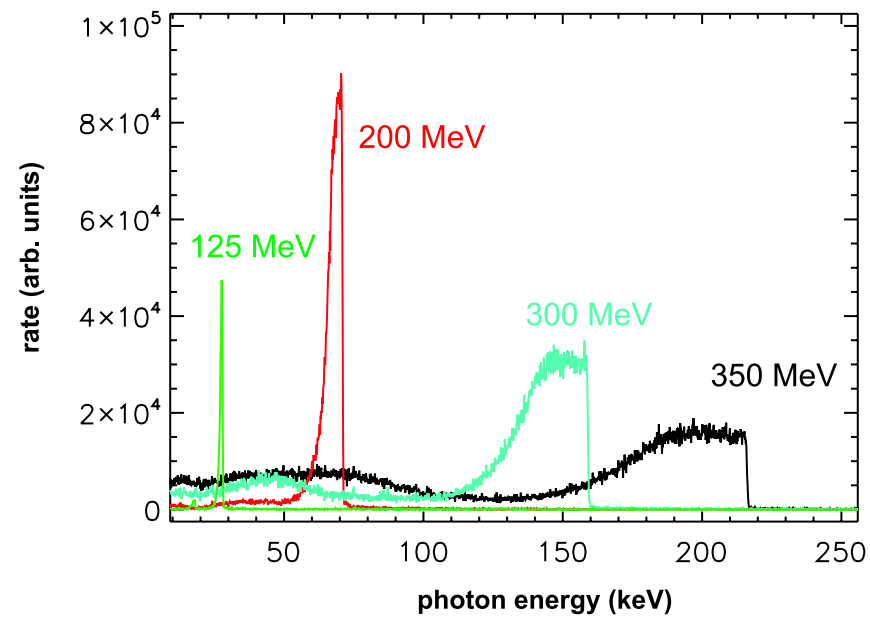

FIG. 6. (Color) Typical spectra of Compton-backscattered photons for low electron beam energies.

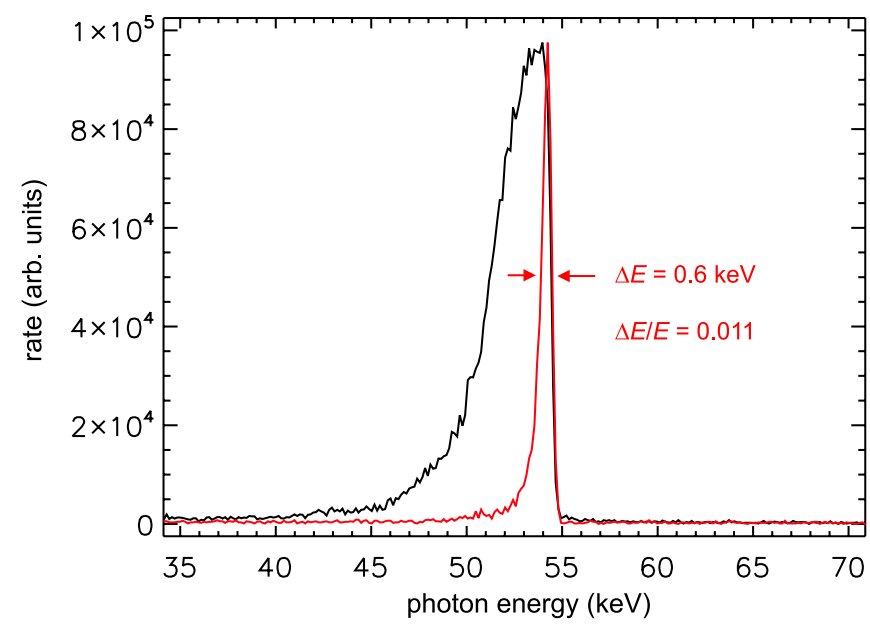

FIG. 7. (Color) Influence of the limitation of the angular acceptance by a collimator on the spectrum of the backscattered photons. With collimation by a tungsten collimator $(r=4 \mathrm{~mm}$ at a distance of approximately $11.8 \mathrm{~m}$ from the middle of the interaction region), quasimonochromatic $\mathrm{x}$ rays can be produced (red curve). The black curve shows the spectrum measured without a collimator. The angular acceptance is then determined by the size of the x-ray detector $(r=12.6 \mathrm{~mm}$, placed at a distance of approximately $12.0 \mathrm{~m}$ from the middle of the interaction region).

the shape of the recorded spectrum. For the fitting procedure the shape without a collimator is more advantageous, whereas for the production of quasimonochromatic x-rays, a collimator can be used to reduce the spectral width.

Based on these measurements, Fig. 8 shows the proportionality factor $p=W / I$ that correlates the electron beam energy $W$ to the bending magnet current $I$. A typical saturation and hysteresis behavior can be seen. The red lines show an analytical approximation for the part with increasing bending magnet currents (up) and decreasing bending magnet currents (down). Either of the analytical

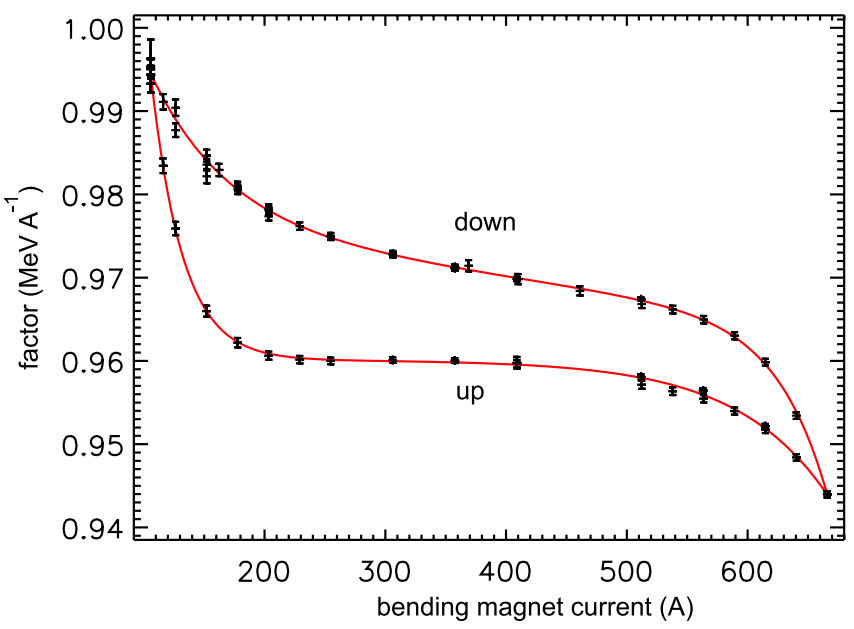

FIG. 8. (Color) Proportionality factor between bending magnet current and electron beam energy. 
TABLE III. Parameters of the approximation for the electron beam energy.

\begin{tabular}{lcccccc}
\hline \hline & $b_{1}$ & $b_{2}$ & $b_{3}$ & $b_{4}$ & $b_{5}$ & $b_{6}$ \\
\hline Down & 0.978 & $-0.205 \times 10^{-4}$ & 0.995 & 0.0156 & 0.944 & 0.0239 \\
Up & 0.960 & $-0.695 \times 10^{-4}$ & 0.995 & 0.0382 & 0.944 & 0.0132 \\
\hline \hline
\end{tabular}

approximations is described as the sum of a straight line and two exponential functions with the parameters $\left(b_{1} \ldots b_{6}\right)$ chosen to have the form

$$
\begin{aligned}
p= & b_{1}+I b_{2}+\left(b_{3}-b_{1}-I_{\mathrm{low}} b_{2}\right) e^{-\left(I-I_{\mathrm{low}}\right) b_{4}} \\
& +\left(b_{5}-b_{1}-I_{\mathrm{high}} b_{2}\right) e^{-\left(I-I_{\mathrm{high}}\right) b_{6}} \text { for } I_{\mathrm{low}} \leq I \leq I_{\mathrm{high}} .
\end{aligned}
$$

In this representation the first exponential describes the saturation behavior for low bending magnet currents and the function approximately has the value $b_{3}$ for the lowest current $I=I_{\text {low }}=105.7 \mathrm{~A}$. The second exponential is attributed to the saturation behavior for large bending magnet currents and the function approximately has the value $b_{5}$ for the maximum bending current of $I=I_{\text {high }}=$ $665.98 \mathrm{~A}$. The parameters $b_{3}$ and $b_{5}$ have the same value for the approximation of the part for increasing or decreasing bending magnet currents. The parameters are given in Table III, the difference in the electron beam energy between measurement and approximation is illustrated in Figs. 9 and 10. The approximation is good enough for most applications and allows the determination of the electron beam energy from the bending magnet current without employing the electron beam energy measurement. The error bars shown in Figs. $8-10$ are the $1 \sigma$ uncertainties of the measurements and are due to calibration errors and counting statistics (typically, the data acquisition time of a photon spectrum is $5 \mathrm{~min}$ ).

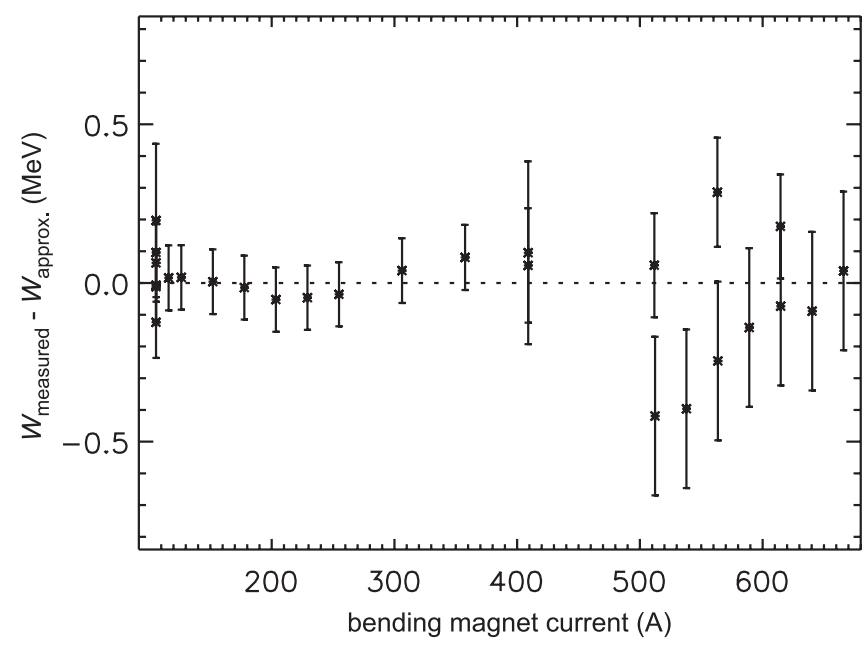

FIG. 9. Difference between measured electron energy and that calculated by the approximation for increasing bending magnet currents.

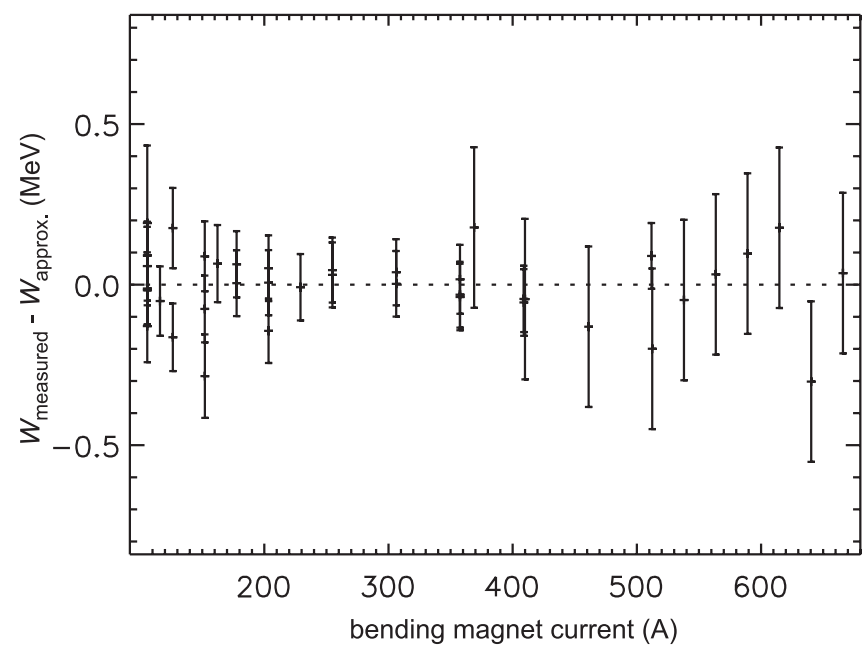

FIG. 10. Difference between measured electron energy and that calculated by the approximation for decreasing bending magnet currents.

\section{B. Electron beam current}

The stored electron beam current can be varied by more than 11 decades from a maximum current of approximately $200 \mathrm{~mA}$ down to one stored electron (1 pA). Currents in the upper range, i.e. above $1 \mathrm{~mA}$, are measured with two commercially available DC parametric current transformers (PCT, Bergoz). For a precise determination of the electron beam current, the offset signal of these monitors has to be taken into account. Figure 11 shows the typical offset values for the two different PCTs, which are depen-

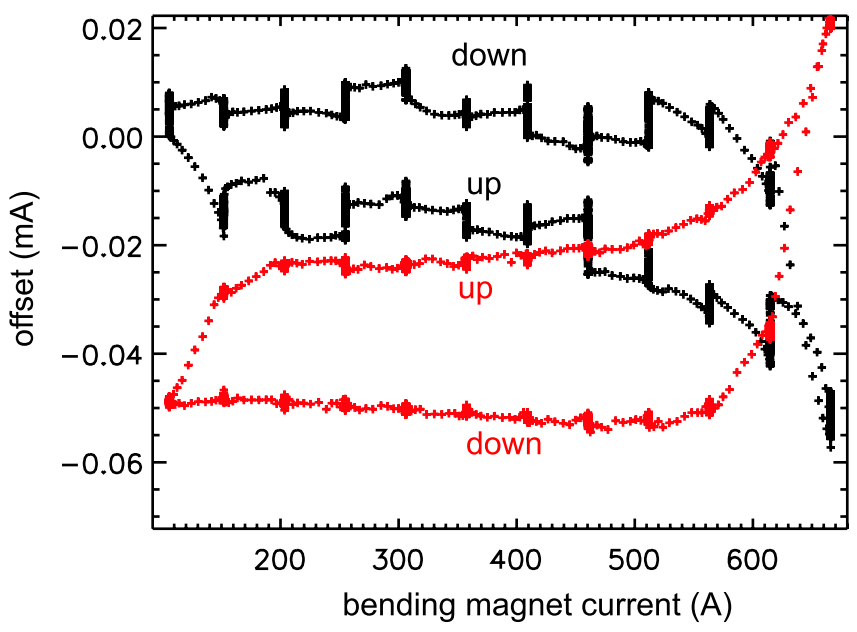

FIG. 11. (Color) The offset currents of the two PCTs (red and black curves) recorded during the increase (up) and decrease (down) of the bending magnet current. The bending magnet current was kept constant at certain values for some time. Because of the oscillating drift of the offset current with time, this results in vertically stacked measurement points of the offset current. 


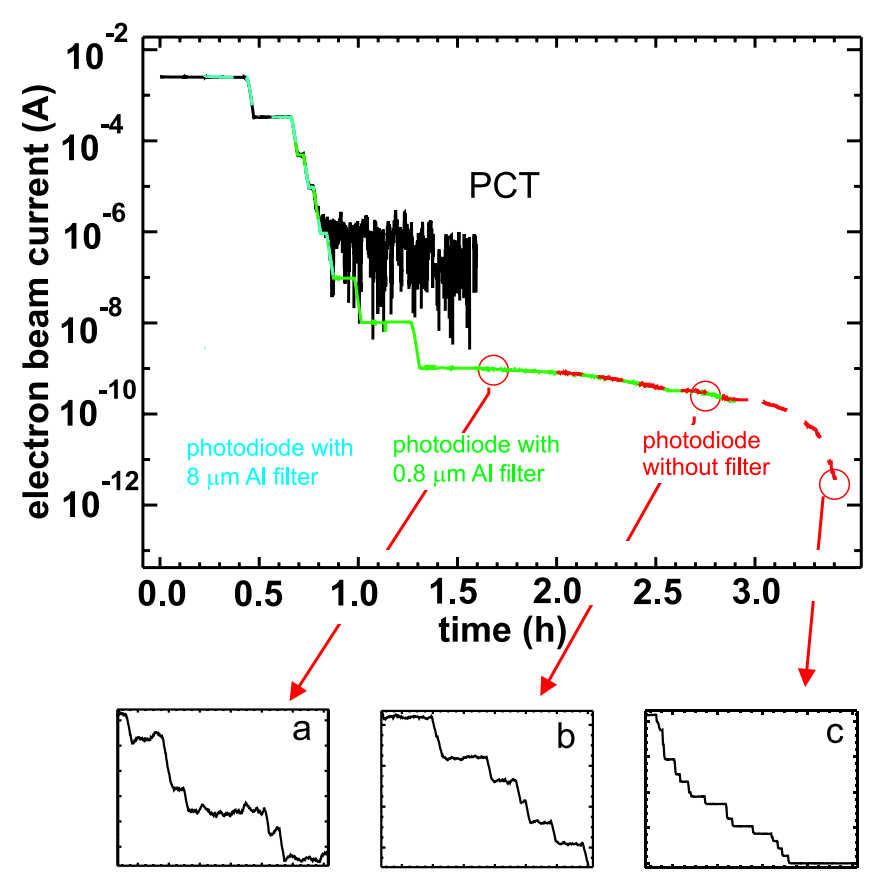

FIG. 12. (Color) Controlled reduction of the electron beam current from some $\mathrm{mA}$ to a single electron.

dent on the electron energy, i.e. the magnetic induction of the surrounding bending magnets.

The relative difference in the readings of the two PCTs differ by less than $2 \times 10^{-4}$ in the current range above $50 \mathrm{~mA}$. Electron currents in the lower range, i.e. below $1 \mathrm{nA}$, are determined by counting the number of stored electrons. For this, the electrons are gradually kicked out of the storage ring by a mechanical scraper that can be moved close to the electron beam, while measuring the steplike drop of the synchrotron radiation intensity by cooled pho-

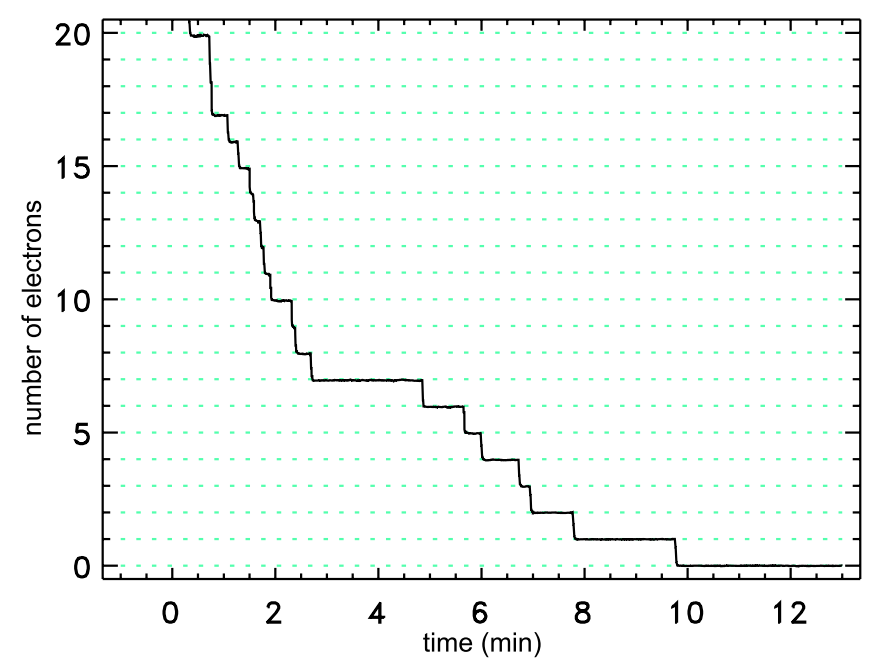

FIG. 13. (Color) A few electrons stored in the MLS. The beam lifetime has artificially been reduced by means of a scraper that is placed close to the electron beam. todiodes. Electron beam currents in the middle range, i.e. from about $100 \mathrm{pA}$ up to $1 \mathrm{~mA}$, are determined by three sets of windowless Si photodiodes with linear response that are illuminated by synchrotron radiation attenuated in intensity in some cases by different filters. The calibration factors of these photodiode-filter combinations, which relate the photocurrent to the electron beam current, are determined by comparison with the electron beam current measured at the upper and lower end of the range as described above. Figure 12 illustrates the reduction of the electron beam current by means of a scraper over the whole range. For currents below $1 \mathrm{nA}$ (1000 electrons, inlay a, in Fig. 12) a steplike decrease becomes visible and is clearly seen around 300 pA (300 electrons, inlay b, in Fig. 12) and below (inlay c, in Fig. 12). Figure 13 shows the measurement of the electron beam current for a few stored electrons. For these measurements, the lifetime of the electron beam, being normally several hours, is artificially reduced by a scraper, which is placed close to the electron beam.

\section{Magnetic induction at the source point}

For the measurement of the magnetic induction at the radiation source point, the bending magnet vacuum chamber is designed in such a way that, after a beam dump, a nuclear magnetic resonance (NMR) probe can be brought to the location of the radiation source point. The magnetic field map of the bending magnet in the area of the radiation source point has been measured [9] and is sufficiently flat so that small displacements of the probe position from the actual source point are tolerable. Figure 14 shows the measured ratio of magnetic induction and bending magnet current. Three different NMR probes have been used to cover the range of the magnetic induction of $0.28 \mathrm{~T}$ up to $1.38 \mathrm{~T}$. The proportionality factor between the bending magnet current and the magnetic induction was also approximated by the sum of a straight line and two exponential functions (see above, red line in Fig. 14). The

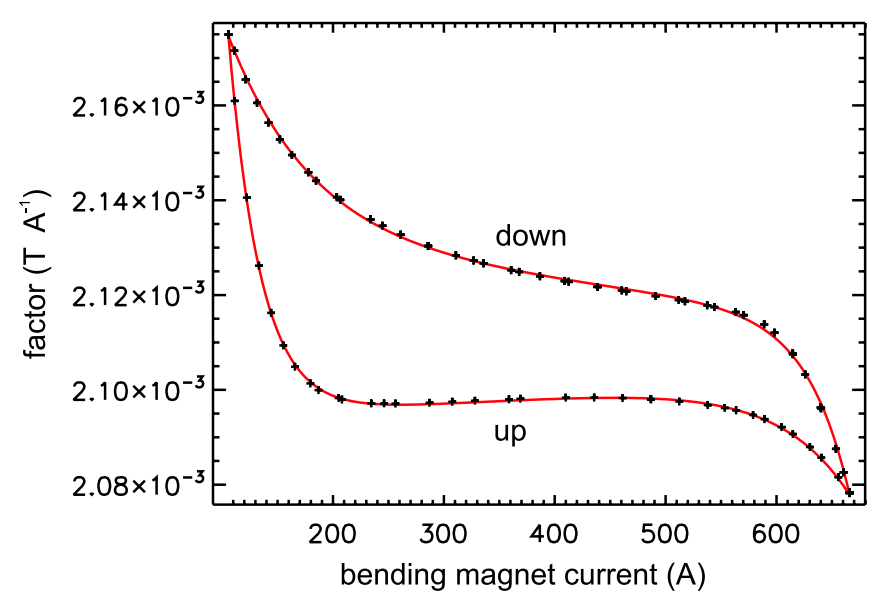

FIG. 14. (Color) Proportionality factor between bending magnet current and magnetic induction. 
TABLE IV. Parameters of the approximation for the magnetic induction at the source point.

\begin{tabular}{lcrrrrr}
\hline \hline & $c_{1}$ & \multicolumn{1}{c}{$c_{2}$} & \multicolumn{1}{c}{$c_{3}$} & $c_{4}$ & $c_{5}$ & $c_{6}$ \\
\hline Down & $2.1338 \times 10^{-3}$ & $-0.27453 \times 10^{-7}$ & $2.1746 \times 10^{-3}$ & $0.13179 \times 10^{-1}$ & $2.0778 \times 10^{-3}$ & $0.26187 \times 10^{-1}$ \\
Up & $2.0929 \times 10^{-3}$ & $0.13934 \times 10^{-7}$ & $2.1746 \times 10^{-3}$ & $0.34538 \times 10^{-1}$ & $2.0778 \times 10^{-3}$ & $0.15524 \times 10^{-1}$ \\
\hline \hline
\end{tabular}

parameters of this approximation are given in Table IV, the relative uncertainty of the approximation is better than $5 \times$ $10^{-4}$ for the whole range and thus sufficient for most applications. If a $1 \times 10^{-4}$ relative uncertainty is required, the magnetic induction has to be measured especially.

\section{Effective vertical source size}

First measurements for the determination of the effective vertical divergence have been performed. A setup for the optical imaging of the electron beam has been put into operation for the determination of the electron beam source size. Figure 15 shows the measured beam size for various electron energies during the ramp up. At low electron beam energies, the beam size is enlarged due to trapped ions. At $630 \mathrm{MeV}$ the vertical size increased due to large coupling. The optical source size measured is estimated to be accurate within $10 \%$. A different setup for the measurement of the vertical source size was applied at another beam line: A
$10 \mathrm{~mm} \times 10 \mathrm{~mm}$ area photodiode with a $8 \mu \mathrm{m} \mathrm{Al} \mathrm{filter}$ was moved vertically through the synchrotron radiation beam at a distance of $2.46 \mathrm{~m}$ from the source. The measured photocurrent was then modeled by calculations with the Schwinger equation, adjusting the parameter for the vertical effective source size to fit the data. Figure 16 shows the measurements (symbols) and the corresponding calculation (lines), for the MLS operated at $630 \mathrm{MeV}$ with large (black) and minimal (red) vertical coupling, yielding an effective source size of 0.8 and $0.25 \mathrm{~mm}$, respectively. The relative accuracy of this approach is estimated to be about $20 \%$, which is sufficiently accurate for most applications.

Both measurements were performed with an electron beam current of $4 \mathrm{~mA}$, but at different positions along the orbit. To compare the results, the values must be scaled with the help of the lattice functions, calculated for the current settings of the MLS. A relative uncertainty of $10 \%$

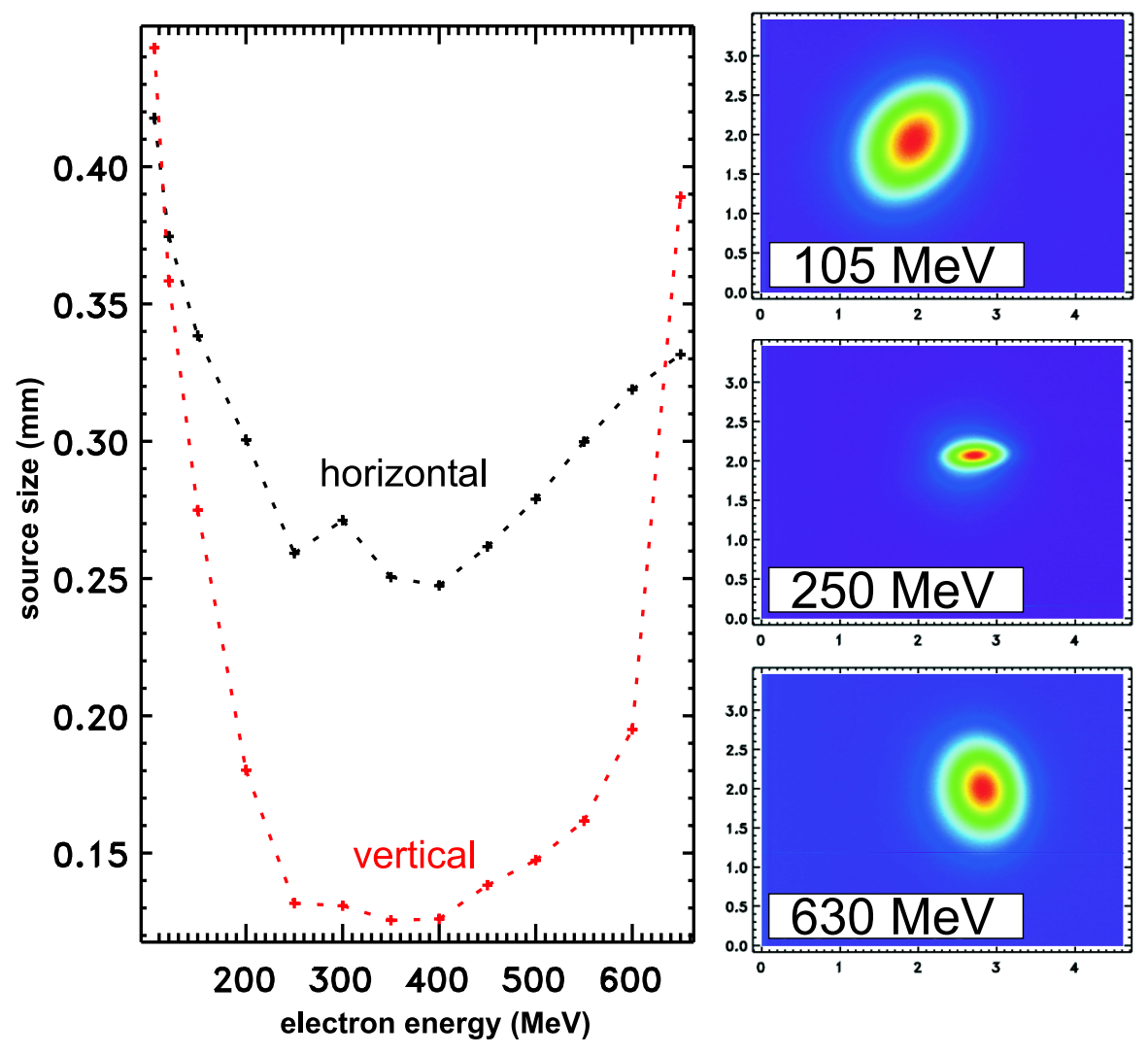

FIG. 15. (Color) Source size $(1 \sigma)$ at $4 \mathrm{~mA}$ electron beam current determined from the optical image of the electron beam. At low electron beam energies, the beam size is enlarged due to trapped ions. At $630 \mathrm{MeV}$ the vertical size increased mainly because of large coupling. 


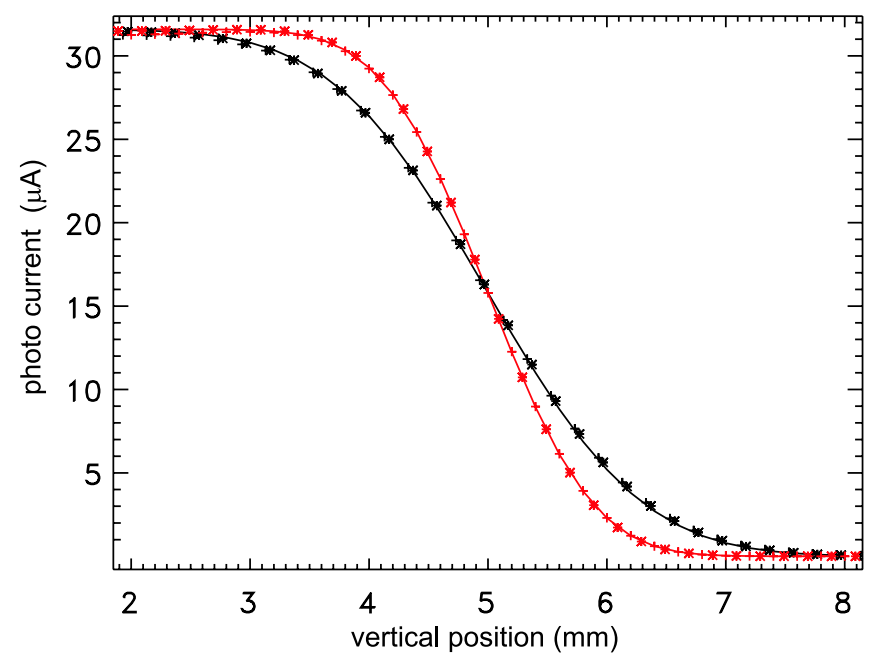

FIG. 16. (Color) Signal of a $10 \mathrm{~mm} \times 10 \mathrm{~mm}$ area photodiode with $8 \mu \mathrm{m} \mathrm{Al}$ filter while being moved vertically into (*) and out of $(+)$ the synchrotron radiation beam. The solid line is the appropriate calculation according to the Schwinger theory. The black and red graphs are for the MLS operated with and without vertically coupled beams at $630 \mathrm{MeV}$, respectively.

in the lattice function calculation is assumed. The value measured with the optical system at a ring energy of $630 \mathrm{MeV}$ and applied vertical coupling was $\sigma_{y}$ (optical) $=$ $(0.39 \pm 0.04) \mathrm{mm}$ (top right measurement point in Fig. 15). The vertical source size scales with the square root of the lattice function $\beta_{y}$. With the given lattice function $\beta_{y}$ for the MLS, this value corresponds to a value of $\sigma_{y}($ optical $\rightarrow$ edge $)=\sigma_{y}($ optical $)\left[\beta_{y}(\right.$ edge $) /$ $\beta_{y}$ (optical) $]^{1 / 2}=(0.62 \pm 0.08) \mathrm{mm}$ at the ring position, where the knife-edge measurement has been performed. The beam divergence and source size are related by the lattice functions $\beta_{y}$ and $\alpha_{y}$ as $\sigma_{y^{\prime}}=\sigma_{y} \sqrt{1+\alpha_{y}^{2}} / \beta_{y}$ and so a corresponding effective source size at a distance of $(2.46 \pm 0.01) \mathrm{m}$ from the source point can be calculated to be $\Sigma_{y}($ optical $\rightarrow$ edge $)=(0.74 \pm 0.10) \mathrm{mm}$. This value is in good agreement with the value determined by the knifeedge measurement at this position of $\Sigma_{y}($ edge $)=(0.80 \pm$ 0.16) mm (black curve in Fig. 16).

\section{E. Geometrical quantities}

The distance to the source point is measured by projecting a fivefold slit into the detection plane. The distance between the slit and the detection plane is known precisely from an interferometric measurement. The distance from the projection plane to the radiation source point at the location of the electron beam can then be calculated from the distance of the projected slits at the detector plane (Fig. 17). An uncertainty of $2 \mathrm{~mm}$ in the determination of the distance to the radiation source point is reached. Typically, at the MLS a detector to be calibrated is placed about $15 \mathrm{~m}$ from the radiation source point, which gives a

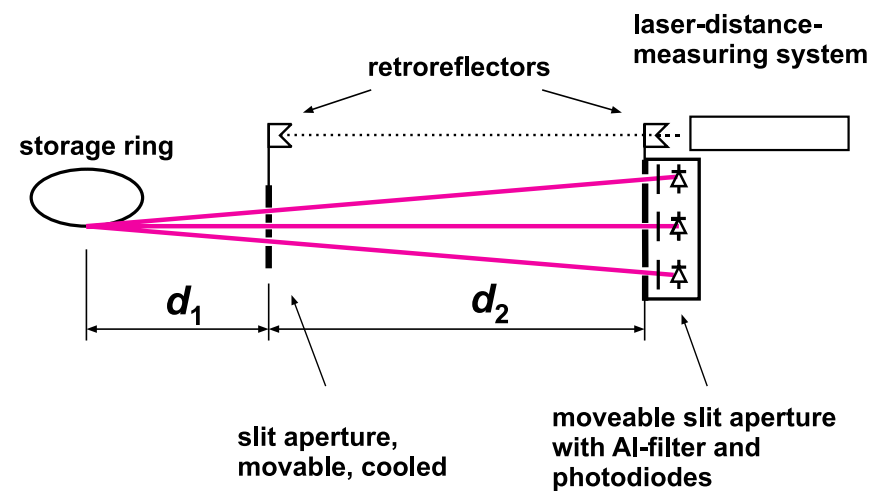

FIG. 17. (Color) Schematic drawing of the setup used to determine the distance to the radiation source point.

relative uncertainty of about $1.3 \times 10^{-4}$ in the determination of the distance.

The vertical emission angle $\psi$ is normally chosen to be zero (measurement in the orbit plane) by adjusting a detector under test in the vertical plane. A typical uncertainty in positioning is $5 \mu \mathrm{rad}$ for a calibration at a $15 \mathrm{~m}$ distance.

The size $a \times b$ of a flux-defining aperture is normally a detector property and not a property of the primary source standard and therefore not included in the discussion of the best measurement capabilities.

\section{SUMMARY}

The setup for the measurement of the parameters for the calculation of the spectral photon flux of the MLS bending magnets has been put into operation successfully. The electron beam energy has been measured over the whole operational range from $105 \mathrm{MeV}$ up to $630 \mathrm{MeV}$ as has been the measurement of the corresponding magnetic induction of the bending magnets. The electron beam current has been set and measured over a dynamic range of more than 11 decades.

The electron beam energy, the magnetic induction at the radiation source point, and the electron beam current can be determined with relative uncertainties below $0.1 \%$. This enables PTB to operate the MLS as a primary source standard from the near IR to the soft x-ray spectral region with an uncertainty below $0.2 \%$ in the calculation of the spectral radiant power, which is very competitive for this spectral region and sufficient for most applications. The MLS therefore perfectly complements the other primary synchrotron radiation sources used by PTB, i.e. a BESSY II bending magnet for the $\mathrm{x}$-ray spectral region and a $7 \mathrm{~T}$ wavelength shifter installed at BESSY II for the hard x-ray spectral region.

[1] G. Ulm, Metrologia 40, S101 (2003).

[2] J. Hollandt, J. Seidel, R. Klein, G. Ulm, M. Ware, and A. Migdall, in Optical Radiometry, edited by A. Parr, R. 
Datla, and J. Garner, Experimental Methods in the Physical Sciences Vol. 41 (Elsevier, New York, 2005), p. 213.

[3] M. Richter, A. Gottwald, F. Scholze, R. Thornagel, and G. Ulm, Adv. Space Res. 37, 265 (2006).

[4] M. Krumrey, M. Gerlach, F. Scholze, and G. Ulm, Nucl. Instrum. Methods Phys. Res., Sect. A 568, 364 (2006).

[5] J. Schwinger, Phys. Rev. 75, 1912 (1949).

[6] R. Thornagel, R. Klein, and G. Ulm, Metrologia 38, 385 (2001).

[7] G. Brandt, J. Eden, R. Fliegauf, A. Gottwald, A. Hoehl, R. Klein, R. Müller, M. Richter, F. Scholze, R. Thornagel, G. Ulm, K. Bürkmann, J. Rahn, and G. Wüstefeld, Nucl. Instrum. Methods Phys. Res., Sect. B 258, 445 (2007).

[8] J. Feikes, M. Abo-Bakr, K. Bürkmann-Gehrlein, M.v. Hartrott, J. Rahn, G. Wüstefeld, R. Klein, and G. Ulm, Proceedings of the 11th European Particle Accelerator Conference, Genoa, 2008 (EPS-AG, Genoa, Italy, 2008), p. 2010.

[9] R. Klein, G. Ulm, P. Budz, K. Bürkmann, J. Rahn, and G. Wüstefeld, Proceedings of the 10th European Particle Accelerator Conference, Edinburgh, Scotland, 2006 (EPSAG, Edinburgh, Scotland, 2006), p. 3314.
[10] R. Klein, G. Brandt, R. Fliegauf, A. Hoehl, R. Müller, R. Thornagel, G. Ulm, M. Abo-Bakr, K. BuerkmannGehrlein, J. Feikes, M. v. Hartrott, K. Holldack, J. Rahn, and G. Wüstefeld, in Proceedings of the 11th European Particle Accelerator Conference, Genoa, 2008, Ref. [8], p. 2055.

[11] R. Klein, G. Brandt, L. Cibik, M. Gerlach, M. Krumrey, P. Müller, G. Ulm, and M. Scheer, Nucl. Instrum. Methods Phys. Res., Sect. A 580, 1536 (2007).

[12] G. Wüstefeld, in Proceedings of the 11th European Particle Accelerator Conference, Genoa, 2008, Ref. [8], p. 26.

[13] R. Müller, A. Hoehl, R. Klein, G. Ulm, M. Abo-Bakr, K. Bürkmann-Gehrlein, J. Feikes, M. v. Hartrott, J. Lee, J. Rahn, U. Schade, and G. Wüstefeld, in Proceedings of the 11th European Particle Accelerator Conference, Genoa, 2008, Ref. [8], p. 2060.

[14] R. Klein, P. Kuske, R. Thornagel, G. Brandt, R. Görgen, and G. Ulm, Nucl. Instrum. Methods Phys. Res., Sect. A 486, 545 (2002).

[15] R. Klein, T. Mayer, P. Kuske, R. Thornagel, and G. Ulm, Nucl. Instrum. Methods Phys. Res., Sect. A 384, 293 (1997). 